\title{
Energy-Saving Management Mechanism Based on Hybrid Energy Supplies for LTE Heterogeneous Networks
}

\author{
Peng Yu, Lei Feng, Wenjing Li, and Xuesong Qiu \\ State Key Laboratory of Networking and Switching Technology, Beijing University of Posts and Telecommunications, \\ Beijing 100876, China
}

Correspondence should be addressed to Peng Yu; yupeng@bupt.edu.cn

Received 5 February 2016; Accepted 29 June 2016

Academic Editor: Gabriel-Miro Muntean

Copyright (C) 2016 Peng Yu et al. This is an open access article distributed under the Creative Commons Attribution License, which permits unrestricted use, distribution, and reproduction in any medium, provided the original work is properly cited.

\begin{abstract}
Aiming at the lack of integrated energy-saving (ES) methods based on hybrid energy supplies in LTE heterogeneous networks, a novel ES management mechanism considering hybrid energy supplies and self-organized network (SON) is proposed. The mechanism firstly constructs ES optimization model with hybrid energy supplies. And then a SON framework is proposed to resolve the model under practical networks. According to the framework, we divide the ES problem into four stages, which are traffic variation prediction, regional Base Station (BS) mode determination, BS-user association, and power supply. And four corresponding low-complexity algorithms are proposed to resolve them. Simulations are taken on under LTE underlay heterogeneous networks. Compared with other algorithms, results show that our mechanism can save $47.4 \%$ energy consumption of the network, while keeping coverage, interference, and service quality above acceptable levels, which takes on great green-economy significance.
\end{abstract}

\section{Introduction}

Recently, energy consumption of Information and Communications Technology (ICT) Industry has an explosive increase, particularly in wireless communication network whose energy consumption occupies a leading position [1]. Since BSs in wireless cellular network consume roughly $60 \%-$ $80 \%$ energy [2], saving energy for BS becomes a research hotspot. With the development of cellular network technologies, LTE heterogeneous networks are commercially deployed step by step, and how to manage BS energy is getting more and more attention as well. Currently, ES approaches for BSs mainly include five categories, which are improvement on hardware components, sleep mode technologies, optimization in radio transmission process, network planning and deployment, and adoption of renewable energy resources [3]. As sleep mode techniques and renewable energy supplies are two research focuses, which do not require equipment upgrade and benefit from low capital costs, so this paper mainly concentrates on these two issues.
For sleep mode techniques, several methods mainly consider energy and throughput tradeoffs [4] or maximizing ES gains [5], which are just for homogeneous networks with similar BS deployments.

Many BS sleep technologies have been proposed for LTE heterogeneous networks as well. And these technologies can be classified into two categories: one is aiming at decreasing power at one time point, and the other is going to save energy during entire time domains. For the first category, BS sleep control is resolved through characterizing energydelay tradeoff [6], dynamic sectorization [7] for cells, or stochastic analysis of optimal Base Station energy [8], and so forth. However, these solutions ignore traffic fluctuations and cannot evaluate temporal ES gains. For the second category, methods in $[9,10]$ analyze temporal and regional ES efficiency of sleep nodes from the point of dynamic traffic reallocation and practical cell topology adjustment, respectively. However, they do not consider coverage and interference control from user perspectives. Additionally, methods in [11, 12] mainly discuss BS sleep gains from the point of traffic prediction 
and BS power mode analysis. But coverage compensation methods and interference control methods for sleep BS are theoretical.

Beyond sleep mode techniques, renewable energy supplies are another approach for saving energy of power grid. In [13], dynamic renewable energy allocation methods with minimal online power are investigated. But BS sleep approach is ignored. And tradeoff and deployment challenges for energy harvesting BS are discussed in [14], but power control is just taking from the streaming perspective, and no detailed methods are given as well. Further, BS sleep method with renewable energy supply is analyzed in [15]; however, it mainly concentrates on mathematical model and solution but does not refer to any management scheme for such green controls.

In fact, to avoid frequent adjustments to the network, ES management (ESM) is defined in self-optimization cases under SON specifications, and it is considered as a popular method to switch off several BSs during low traffic period [16]. Based on our previous work on BS sleep methods with coverage and interference consideration [17] and hybrid energy supplies [18], we propose an ES management mechanism based on hybrid energy supplies for LTE heterogeneous network. This mechanism uses self-organized architecture and combines BS sleep technology and renewable energy supply together. The contributions are as follows.

Firstly, by analyzing resource allocation, Quality of Service (QoS), and energy consumption in LTE heterogeneous network, we build an ES optimization model based on hybrid energy supplies in time domain. The model fully considers constraints of power, interference, coverage, and resource and has strong universality.

Then, to make the ES model be resolved under practical network, we propose a self-organized framework which defines the management architecture and ES procedures. In addition, in order to reduce the computational complexity, the model is divided into four stages, which are traffic variation monitoring, regional BS mode determination, BSuser association, and power supply.

To address these four problems, we design S-ARIMA based traffic prediction algorithm, low-complexity BS cooperation algorithm based on geographical topology, distributed user allocation algorithm, and sustainable power supply algorithm, respectively. Finally, the simulations are conducted in LTE underlay heterogeneous network. Results show that the mechanism can obtain more regional ES gains compared to other algorithms, while maintaining user coverage, interference, and QoS above acceptable levels.

The rest of the paper is organized as follows. Section 2 presents ES optimization model in LTE heterogeneous network, which develops resource allocation and BS energy consumption model, and three key points for resolving the model are analyzed then. Section 3 gives the self-organized framework for ESM, which includes the management architecture, the management entities, and management procedures. Based on the framework, Section 4 proposes corresponding Seasonal Auto-Regressive Integrated Moving Average (SARIMA) based traffic prediction algorithm, BS cooperation algorithms based on geographical topology, distributed user allocation algorithm, and sustainable power supply algorithm with complexity analysis. Section 5 numerically evaluates the mechanism and algorithm. Conclusions and future work are given in the last section.

\section{ES Optimization Model}

For ES optimization model, this paper primary focuses on resource allocation method and BS energy consumption model and constructs an optimization model based on hybrid energy supplies. Moreover, key points to resolve the problem will be discussed as well.

2.1. Resource Allocation Method. Assuming that there are $N$ BSs in underlay LTE heterogeneous network, including $N_{1}$ macro BSs (eNodeB) and $N_{2}$ micro BSs (microcell), each eNodeB contains three sectors (except for those on the boundary). $\mathbf{B}_{M}$ and $\mathbf{B}_{m}$ denote the set of eNodeB and the set of microcells, and $\mathbf{B}=\mathbf{B}_{M} \cup \mathbf{B}_{m}=\left\{B_{j}\right\}$. The network has $K$ kinds of services. Supposing that time domain is $[0, T]$, for $B_{j}$ and user $u_{i}, \mathbf{M}_{j}(t)$ is the set of active users of $B_{j}$ at time $t$. Assume that $\mathbf{X}(t)=\left\{x_{i j}(t)\right\}$ is the BS association matrix, where $x_{i j}(t)=1$, if user $u_{i}$ is served by $B_{j}$; otherwise $x_{i j}(t)=0$. Then Signal to Interference plus Noise Ratio (SINR) experienced by user $u_{i}$ from $B_{j}$ at time $t$ is given by

$$
\gamma_{i j}(t)= \begin{cases}\frac{p_{i j}(t) \cdot g_{i j}(t)}{\mathcal{N}_{0}+\mathscr{I}_{i}(t)+I_{i j}(t)}, & x_{i j}(t)=1 \\ 0, & x_{i j}(t)=0,\end{cases}
$$

where $p_{i j}(t)$ denotes the power for each resource block (RB) and $\mathbf{P}(t)=\left\{p_{i j}(t)\right\}$ is defined as its matrix. $g_{i j}(t)$ denotes the channel gain between $B_{j}$ and user $u_{i}$. $\mathcal{N}_{0}$ denotes the thermal noise. $\mathscr{I}_{i}(t)$ and $I_{i j}(t)$ denote interference outside $B_{j}$ and inside $B_{j}$ for user $u_{i}$, respectively.

Assuming that AMC (Adaptive Modulation and Coding) is used, then spectral efficiency $\varphi_{i j}(t)(\mathrm{bps} / \mathrm{Hz})$ when user $u_{i}$ is served by $B_{j}$ is given by [7]

$$
\varphi_{i j}(t)= \begin{cases}0, & \gamma_{i j}(t)<\gamma_{\min }, \\ \xi \log _{2}\left(1+\gamma_{i j}(t)\right), & \gamma_{\min } \leq \gamma_{i j}(t)<\gamma_{\max }, \\ \varphi_{\max }, & \gamma_{i j}(t) \geq \gamma_{\max },\end{cases}
$$

where $\gamma_{\min }$ is the minimum SINR and $\xi \in[0,1]$ is the attenuation factor. $\gamma_{\max }$ and $\varphi_{\max }$ are the maximum of SINR and spectral efficiency, respectively. Further, we can derive the number of RBs for user $u_{i}$ required from $B_{j}$ as

$$
\beta_{i j}(t)=\left\lceil\frac{r_{i j}(t)}{W_{\mathrm{RB}} \varphi_{i j}(t)}\right\rceil,
$$

where $r_{i j}(t)$ is the required service rate from serving $B_{j}$ to user $u_{i}$ and $W_{\mathrm{RB}}$ is the bandwidth for each $\mathrm{RB}$. Then load factor $L_{j}(t)$ of BS can be defined as

$$
L_{j}(t)=\frac{1}{\beta_{j}^{T}} \sum_{i=1}^{\left|\mathbf{M}_{j}(t)\right|} \beta_{i j}(t),
$$


where $\beta_{j}^{T}$ represents the total number of RBs of BS $j$. Load factor can be used as one important indicator to compute BS dynamic power.

2.2. BS Energy Consumption Model. For $B_{j}$, assume that its transmit power is $P_{j}^{T}(t)$ at time $t$ and maximum operational power is $P_{j}^{M}$. And ratio of BS static power to $P_{j}^{M}$ is denoted as $\delta_{j}$. Then the power of BS $B_{j}$ at time $t$ can be expressed as

$$
\begin{aligned}
& P_{j}(t)=\left(1-\delta_{j}\right) L_{j}(t) P_{j}^{M}+\phi_{j}(t) \delta_{j} P_{j}^{M}, \\
& \phi_{j}(t)= \begin{cases}1, & L_{j}(t)>0, \\
\frac{\varepsilon}{\delta_{j}}, & L_{j}(t)=0,\end{cases}
\end{aligned}
$$

where $\varepsilon$ denotes energy proportion of sleep BSs to maintain basic management functions relative to maximal operational power. With (5), we can evaluate BS power in different modes.

For hybrid energy supplies, we assume that each outdoor eNodeB has a panel powered by renewable energy such as wind energy and solar energy. Still, primary energy of panel of $B_{j}$ is $E_{j}^{0}(\mathrm{KWH})$ and power generation rate is $v_{j}(t)$. To ensure system stability, renewable energy is used according to the power level of eNodeB. Therefore, at time $t$, energy generation rate $e_{j}(t)$ and stored energy $E_{j}(t)$ of each panel can be expressed as

$$
\begin{aligned}
& e_{j}(t)=f\left(v_{j}(t) ; P_{j}(t)\right), \\
& E_{j}(t)=E_{j}^{0}+\int_{0}^{t} e_{j}(t) d t .
\end{aligned}
$$

In (7), function $f(\cdot)$ is determined by the power supply policy. Assuming that all derived energy of eNodeBs can be stored into the battery, then required power $a_{j}(t)$ from power grid and corresponding consumed energy $A_{j}(t)$ can be written as

$$
\begin{aligned}
& a_{j}(t)=h\left(v_{j}(t) ; P_{j}(t)\right), \\
& A_{j}(t)=\int_{0}^{t} a_{j}(t) d t .
\end{aligned}
$$

In (8), function $h(\cdot)$ is still determined by the power supply policy. According to (6)-(9), we can obtain accurate value of energy consumption in power grid and renewable energy system.

After determining resource allocation method and BS energy consumption model, we need to select a reasonable optimization model and solve it with proper algorithms as shown in the following sections.

2.3. ES Optimization Model. In this paper, our target is to minimize energy consumption from power grid and minimize the stored energy in each battery, while ensuring coverage, interference, and QoS in whole region. Therefore, for all users $u_{i} \in \mathbf{M}_{j}(t)$ and BSs $B_{j} \in \mathbf{B}$, the optimization model can be expressed as (10).
As shown in (10), the optimization objects are association matrix $\mathbf{X}(t)$ and allocated power matrix $\mathbf{P}(t)$ at time $t$. The first is service quality constraint, where $P_{j k}^{B}(t)$ is the service blocking probability for service $k$ at $\mathrm{BS} j$ and $P_{T}^{B}$ is the threshold of service blocking probability for each service. The second constraint makes sure that one user can only be served by no more than one BS simultaneously. The third constraint is used to guarantee that RB number of each BS can satisfy user demand. The next constraint is the restriction for transmit power with control factor $\alpha$. The fifth constraint is related to the signal strength, where $\sigma_{i j}(t)=p_{i j}(t) \cdot g_{i j}(t)$ denotes the signal strength received by user $u_{i}$ from $B_{j}$ at time $t$. Here $\operatorname{Pr}(x)$ means accumulative probability for condition $x$. It ensures that the accumulative probability of received signal strength for active users (above threshold $\chi_{\text {min }}$ ) is higher than $P_{\sigma}$. The sixth constraint is involved with regional interference. That is, the accumulative probability of SINR received by users from serving BS (above threshold $\gamma_{\min }$ ) is limited by the predefined target $P_{\gamma}$. Consider

$$
\begin{aligned}
\mathbb{P}: \min _{\mathbf{X}(t), \mathbf{P}(t)} & \sum_{j \in \mathbf{B}_{M}} E_{j}(T)+\sum_{j \in \mathbf{B}_{M}} A_{j}(T), \\
\text { s.t. } \quad & P_{j k}^{B}(t) \leq P_{T}^{B}, \quad \forall j, k, t, \\
& \sum_{j=1}^{N} x_{i j}(t)=1, \quad \forall i, t, \\
& \left|\mathbf{M}_{j}(t)\right| \\
& \sum_{i=1} \beta_{i j}(t) \leq \beta_{T}, \quad \forall j, t, \\
& \left|\mathbf{M}_{j}(t)\right| \\
& \sum_{i=1} \beta_{i j}(t) p_{i j}(t) \leq \alpha P_{j}^{T}(t), \quad \forall j, t, \\
& \operatorname{Pr}\left(\sigma_{i j}(t) \geq \chi_{\min } x_{i j}(t)\right) \geq P_{\sigma}, \quad \forall t, \\
& \operatorname{Pr}\left(\gamma_{i j}(t) \geq \gamma_{\min } x_{i j}(t)\right) \geq P_{\gamma}, \quad \forall t .
\end{aligned}
$$

In this optimization model, if we want to minimize $A_{j}(T)$ and $E_{j}(T)$ at the same time, we should consider ES schemes from two points: one is maximizing power supply of renewable energy with proper power scheduling, and the second is minimal BS operational power with BS sleep strategy. Due to the complexity of the optimization model and practical limits of cellular networks, we will analyze the key points for resolving the model.

2.4. Key Points for Resolving the Model. Since the model contains a lot of constraints, so much information should be collected from the network to figure out whether these conditions are satisfied. Still, resolutions with $\mathbf{X}(t)$ and $\mathbf{P}(t)$ require network control to change wireless parameter for BSs and reconnection for users; all these actions require the help of network management functions. Currently, most network management work is done manually. However, ES action always requires frequent adjustments which may put heavy burdens on network. So traditional manual control 


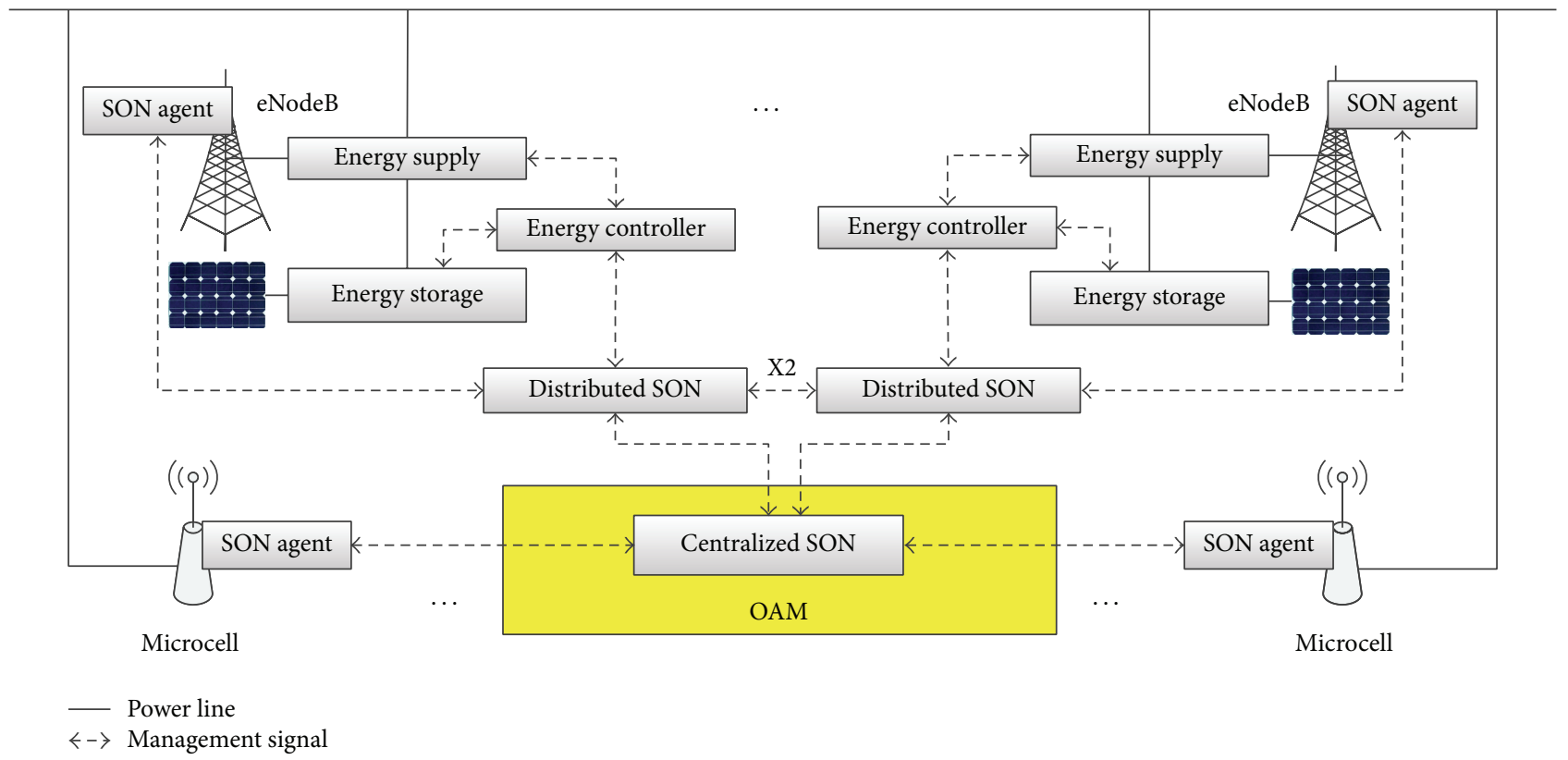

FIGURE 1: Illustration of compensation under irregular scenario.

may be high cost. To make ES action be executed more efficiently, ESM defined by 3GPP in SON use cases [16] and self-organized BS cooperation method [19] will be adopted in this paper as a suitable management policy and compensation method. As optimization objects are discrete matrix and continuous matrix and the constraints are nonlinear, from [7], we can derive that this problem is a nonconvex optimization problem and is hard to be resolved. To make the optimization model be executed under practical networks, we should take the following four points with low-complexity methods into considerations.

\subsubsection{How to Determine When ES Actions Can Be Executed?} ES actions can be carried out through BS sleep and corresponding BS parameter adjustments (such as power, tilt, and neighbor relationship). ES model considers ES problems during the whole time domain, but we could not execute it at each time point so as to avoid frequent parameter adjustments. Therefore, execution frequency should be proper. Still, as ES actions is always triggered by the traffic variations, accurate traffic prediction method is profitable as well.

2.4.2. How to Determine the BS Mode When ES Actions Are Executed? During ES period, several BSs will be slept. On one hand, we want to sleep as more BSs as possible. On the other hand, regional coverage and traffic should be accommodated by the active BSs, so geographic BS deployment and regional traffic load should be considered as well. Thus we should find an efficient BS mode determination method.

2.4.3. How to Keep Users' QoS during ES Period? As BS sleep will change the network topology and no doubt affect user QoS such as perceived signal strength, interference level, and service blocking probability, we should give a method to adjustment parameters from user QoS perspective thus to guarantee regional user QoS and network performance above acceptable level. As parameters are the same to each user, the method should leverage the parameter effect among BSs and users.

2.4.4. How to Maximize the Utilization of Renewable Energy Supplies? As renewable energy supplies come from the solar energy or wind energy which vary drastically along with environment, the energy generation rate will change along with time as well. However, renewable energy takes on the best green benefits, so a method should be given to maximize the utilization of renewable energy supplies. Moreover, the method should guarantee that power supply for each eNodeB is stable and sustainable.

Aiming at resolving above key points, we propose a selforganized framework to address them, which will be shown in Section 3.

\section{Self-Organized Framework for ESM}

According to the ESM definition and the scenarios of LTE heterogeneous networks with hybrid supplies, we give the self-organized framework for ESM in Figure 1.

As shown in Figure 1, to make ESM more practical, we should consider the management architecture, the management entities, and management procedures in SON framework.

3.1. Management Architecture. There are three kinds of management architecture in SON, which are centralized SON, distributed SON, and hybrid SON. Considering both distributed massive BSs and the OAM regional function, we use hybrid SON here, as shown in Figure 1. 


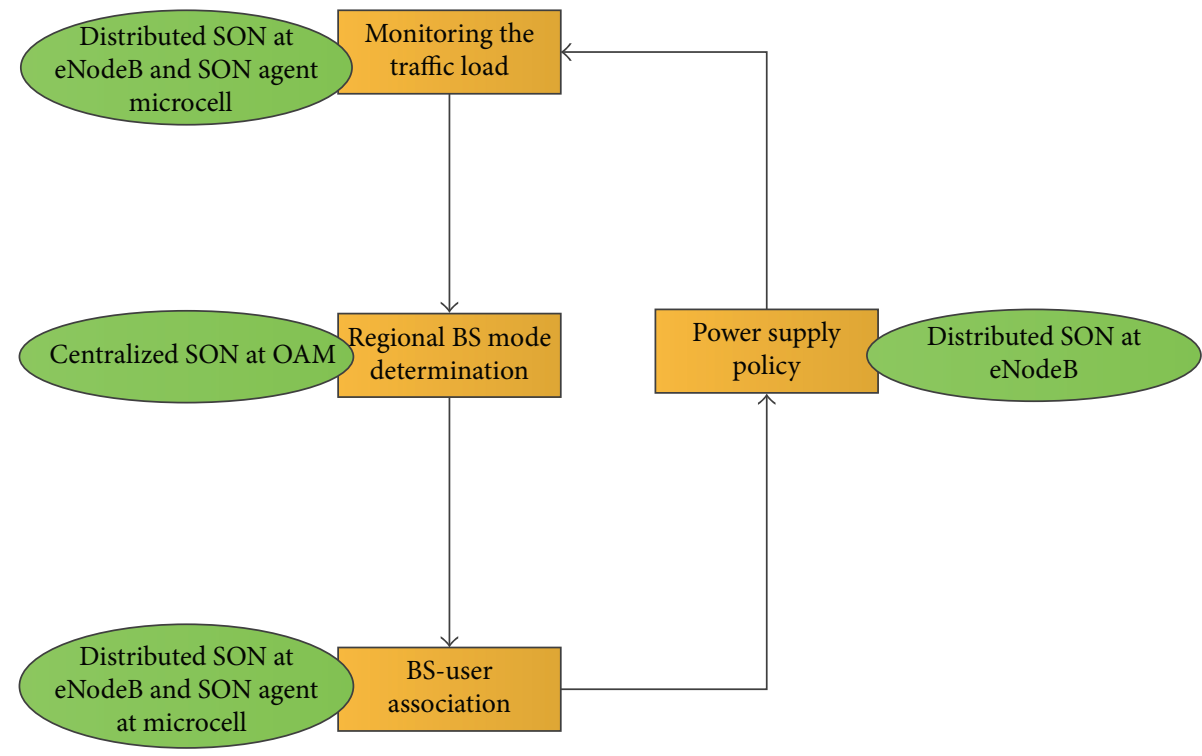

FIGURE 2: Procedures of ESM.

The hybrid management architecture includes centralized SON and distributed SON. Here we assume that distributed SON communicates with SON agents deployed on each eNodeB. Distributed SON is responsible for guaranteeing users' QoS under each eNodeB. Still, distributed SON communicate with each other through X2 interface.

Moreover, centralized SON is deployed on Operation, Administration, and Maintenance (OAM) system to manage regional information, such as network topology and regional traffic load. Centralized SON communicates with distributed SON at each eNodeB and SON agent at each microcell. Regional control algorithms will be executed by centralized SON as well.

3.2. Management Entities. As shown in Figure 1, to keep fluent management among different network elements (including battery, eNodeB, and microcell), we should set the following management entities:

(1) SON agent at each eNodeB, which monitors the basic parameters, power, key performance indicators, and traffic data, and pushes the ES actions from distributed SON to eNodeB.

(2) SON agent at microcell, which monitors the basic parameters, power, key performance indicators, and traffic data, and pushes the ES actions from centralized SON to microcell.

(3) Centralized SON at OAM system, which collects regional information from the eNodeB and microcells and gives regional ES action suggestions.

(4) Distributed SON at each eNodeB, which collects and stores the information of the entire eNodeB and energy controller and gives local ES action suggestion for users under current eNodeB.

(5) Energy supply, which controls the energy supply for each eNodeB under hybrid energy sources.
(6) Energy storage, which stores the energy coming from the renewable energy sources and power lines.

(7) Energy controller, which determines the energy supply method according to the energy storage, eNodeB power requirements, and the control information from the distributed SON.

With above entities, we can obtain effective energy supply and energy saving with hybrid management architecture.

3.3. Management Procedures. As ESM is a looped control for the whole network, next we will give the procedures for how to implement it. With the management architecture and management entities, the procedures are shown in Figure 2.

As shown in Figure 2, our procedures have four stages under the management of different SONs:

(1) For distributed SON at each eNodeB and SON agent at each microcell, they monitor the traffic load of each eNodeB and each microcell and predicate the traffic variations for the next period. From [7], we can assume that traffic per hour is unchanged, which makes it possible to take the traffic prediction only from each hour.

(2) According to traffic prediction results and regional network information, the centralized SON determines the BS mode and traffic accommodation policies. The determination should make sure that no coverage hole exists in the network as well. Here we set $s_{j}(t)$ as state of $B_{j}$ at time $t$ corresponding to the mode (i.e., $s_{j}(t)=0$ as sleep mode and $s_{j}(t)=1$ as active mode).

(3) After BS mode determination, next distributed SON at eNodeB and SON agent at microcell should find appropriate way to adjust parameters at each BS thus to make each user associate with proper BS above acceptable QoS levels. 
(4) Once the parameter adjustments are determined, we can obtain the power required for each BS. Then, distributed SON at each eNodeB determines the power supply policies to maximize the utilization for renewable energy.

After the energy supply policies are determined, all the parameter adjustments and power supply strategies will be executed by each BS under the control of SON agent. And then the whole network will return back to the traffic monitoring stages.

Above procedures just denote what to do for different SON entities. We should give the proper algorithms for different stages as well. To resolve the key points in the ESM procedures, we proposed S-ARIMA based traffic prediction algorithms, BS cooperation algorithm based on geographic topology, distribution user allocation algorithm, and sustainable power supply algorithm to resolve them.

\section{Corresponding Practical Algorithm}

4.1. S-ARIMA Based Traffic Prediction Algorithm. To judge when ES actions can be triggered or rolled back, we should know how traffic will be changed along with time. Here traffic is taken as the load factor in (4). There are many traffic prediction methods which have been used in BS sleep methods, such as Holt-Winters in [11] and online stochastic game theoretic algorithm in [20]. But they are not suitable for traffic with small value and the accuracy can be improved. In this paper, according to the periodic features of traffic, we use S-ARIMA traffic prediction algorithm to estimate the future traffic.

The S-ARIMA model is given by

$$
\begin{aligned}
\phi_{x} & (C) \Phi_{X}\left(C^{y}\right)(1-C)^{d}\left(1-C^{y}\right)^{D} \dot{L}(t) \\
& =\theta_{q}(C) \Theta_{Q}\left(C^{y}\right) \rho(t),
\end{aligned}
$$

where

$$
\dot{L}(t)= \begin{cases}L(t)-\mu, & d=D=0, \\ L(t), & \text { others }\end{cases}
$$

with

$$
\begin{gathered}
\phi_{x}(C)=1-\phi_{1} C-\phi_{2} C^{2}-\cdots-\phi_{x} C^{x} \\
\Phi_{X}\left(C^{y}\right)=1-\Phi_{1} C^{y}-\Phi_{2} C^{2 y}-\cdots-\Phi_{X} C^{X y}, \\
\theta_{q}(C)=1-\theta_{1} C-\theta_{2} C^{2}-\cdots-\theta_{q} C^{q} \\
\Theta_{Q}\left(C^{y}\right)=1-\Theta_{1} C^{y}-\Theta_{2} C^{2 y}-\cdots-\Theta_{Q} C^{Q y} .
\end{gathered}
$$

In (11) (13), $\phi_{x}(C)$ and $\theta_{q}(C)$ are the conventional autoregression operator and moving average operator, respectively. Correspondingly, $\Phi_{X}\left(C^{y}\right)$ and $\Theta_{Q}\left(C^{y}\right)$ are seasonal autoregression operator and moving average operator. $\rho(t)$ is the white noise with zero average and $\mu$ is a constant value. $C$ is the backward shift operator as $C L(t)=L(t-1) . L(t)$ is the load factor of BS and is taken as the time sequences.
Moreover, $d$ and $D$ are differential order and seasonal differential order, respectively. Then we call the model in (11) $\operatorname{S-ARIMA}(x, d, q) \times(X, D, Q)$, model with season $y$.

To obtain the proper S-ARIMA model for time sequences, we should execute the following steps.

Step 1. Compute the differences $\nabla$ and seasonal differences $\nabla_{y}$ to obtain stationary series for the given time sequences.

Step 2. Compute the Autocorrelation Function (ACF) and Partial Autocorrelation Function (PACF) for the stationary sequences, and then match them to known values in $\mathrm{S}$ ARIMA model. If more than one combination of $(x, d, q) \times$ $(X, D, Q)$ is proper, we then adopt the one with minimal Akaike's Information Criterion (AIC) as the tentative model.

Step 3. Compute the initial estimation for model parameters in $\operatorname{S-ARIMA}(x, d, q) \times(X, D, Q)$, with Maximum Likelihood Estimation (MLE) or moment estimation.

Step 4. After fitting, check whether the residual sequences can be considered as white noise with ACF and PACF computation. If the checking is not passed, improvement for the parameters will be given, and fitting and checking procedures will be executed until the checking is passed.

As traffic variations in each BS take on obvious seasonable feature, S-ARIMA is an effective prediction algorithm for cellular traffic.

In fact, as time series prediction models require lots of computations and iterations, their computational complexities are determined by data volume, the number of parameters, the estimation method, and time cycle. So, it is hard to give an accurate mathematical expression for time complexity. However, many tools such as RStudio have integrated S-ARIMA model into them, and it is easy to use this tool to predicate the time sequences.

4.2. BS Cooperation Algorithm Based on Geographic Topology (BCAGT). For eNodeB, since static power of each BS occupies more than its $50 \%$ energy consumption as described in [21], so the target of this part is to maximize number of sleep BSs with global information at the centralized SON. In addition, three constraints should be taken into account:

(i) After sleeping BSs and reallocating traffic load, no active node is overloaded.

(ii) To reduce effect of frequent handovers caused by BS sleeping, number of sleep times per BS during entire time domain cannot exceed a threshold (e.g., 1 time).

(iii) To ensure satisfactory coverage, each sleep BS has at least one active neighbor BS.

With above considerations, BCAGT which mainly use the network topology information can be obtained beforehand.

For slept BSs, one, two, or three neighbor BSs can cooperate to compensate coverage and capacity [22], as illustrated in Figure 3. Micro BS $B_{12}$ is fully compensated by Macro BS $B_{11}$ which is called EP (Entire Pair) of $B_{12}$. Additionally, macro 


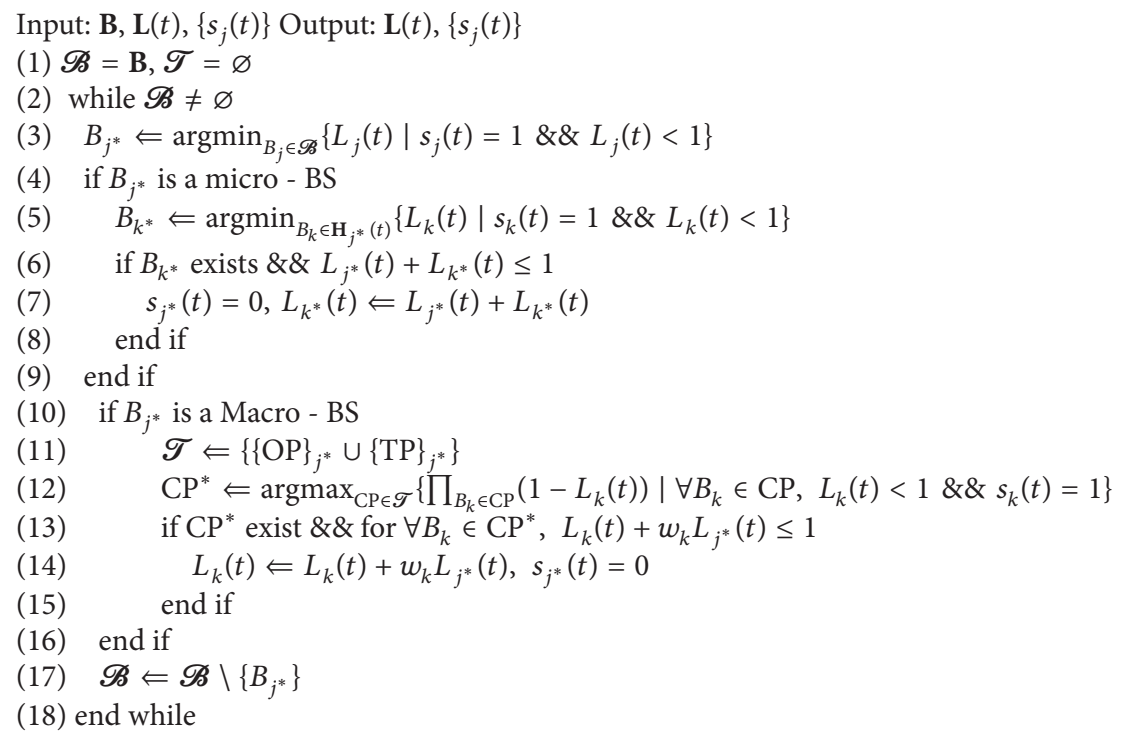

Algorithm 1: Description of BS cooperation algorithm.

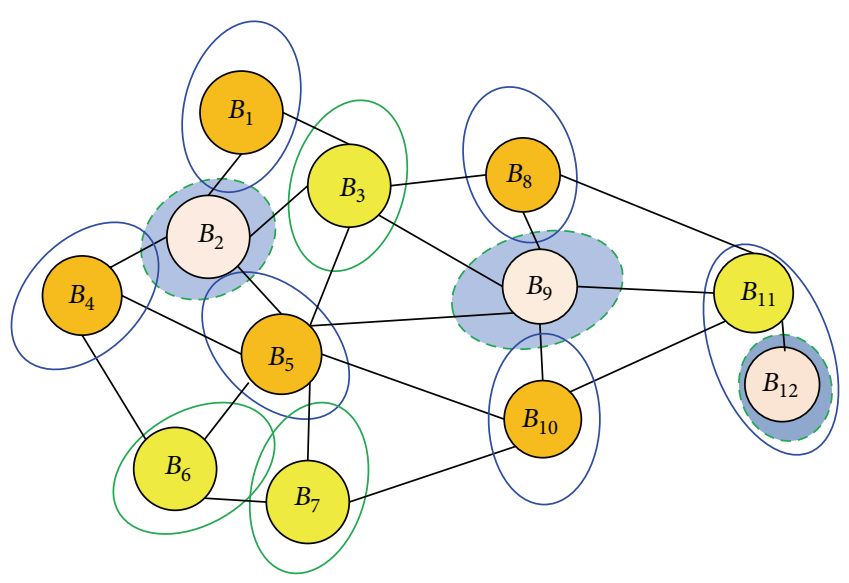

FIGURE 3: Illustration of compensation under irregular scenario.

BS $B_{9}$ can be compensated by macro BS opposite pair (OP) $\left(B_{8}, B_{10}\right)$, and macro BS $B_{2}$ can be compensated by macro BS trigonal pair (TP) $\left(B_{1}, B_{4}\right.$, and $\left.B_{5}\right)$. The definitions of OP and $\mathrm{TP}$ can be seen in our previous work in $[23,24]$.

Based on definitions of OP and TP, the time domain $[0, T]$ can be divided into four phases due to regional traffic states [17]. In peak and midnight phase, the states of BSs remain the same. And in traffic decreasing phase, this algorithm should be executed at the beginning of each hour. The process is shown as follows in Algorithm 1. This algorithm shows the process of sleep BS selection with load decline. Similarly, based on symmetry of load variation in time domain, the reverse process of BCAGT is used to recover sleep BSs during traffic increasing phase. The four phases are determined according to the fitting for historic traffic load. Moreover, the traffic load used in this algorithm is the prediction traffic load as well.
Here $\mathbf{L}(t)$ is the traffic prediction vector for regional BSs. As shown in Algorithm 1, firstly, we find the active $B_{j^{*}}$ with the lowest load. If $B_{j^{*}}$ is a micro BS, select the active BS $B_{k^{*}}$ with the lowest load from its neighbor macro BS set $\mathbf{H}_{j^{*}}(t)$ which can completely cover $B_{j^{*}}$. If $B_{k^{*}}$ exists and is able to absorb the load of $B_{j^{*}}$, then we can transfer the load to $B_{k^{*}}$ and sleep $B_{j^{*}}$. If $B_{j^{*}}$ is a macro BS, its OP set $\{\mathrm{OP}\}_{j^{*}}$ and TP set $\{\mathrm{TP}\}_{j^{*}}$ should be selected to form the set of compensation elements denoted as $\mathscr{T}$. Then select compensation element $\mathrm{CP}^{*}$ which satisfies the conditions that $\forall B_{k} \in \mathrm{CP}^{*}$ is active and not overloaded, and the product of surplus load of all BSs in $\mathrm{CP}^{*}$ is maximum. If $\mathrm{CP}^{*}$ exists and is able to absorb the load of $B_{j^{*}}$, its load will be allocated by a ratio of $w_{k}$ to BSs in $\mathrm{CP}^{*}$, and then we can sleep it. According to [20], $w_{k}$ is defined as

$$
w_{k}=\frac{\ell_{k j^{*}}^{2}}{\sum_{i \in \mathrm{CP}^{*}} \ell_{i j^{*}}^{2}} .
$$

Here $\ell_{i j}$ is the distance from BS $i$ to BS $j$.

After selecting $B_{j^{*}}$, all BSs in this region should be traversed until all BSs are analyzed. We can easily find that complexity of Algorithm 1 is $O\left(\left|\mathbf{B}_{m}\right| \cdot \max \left\{\mathbf{H}_{j^{*}}(t)\right\}+\right.$ $\left.\left|\mathbf{B}_{M}\right| \cdot \max \left\{\left|\{\mathrm{OP}\}_{j^{*}}\right|,\left|\{\mathrm{TP}\}_{j^{*}}\right|\right\}\right)$. Based on analysis from [17], we know that $\max \left\{\left|\{\mathrm{OP}\}_{j^{*}}\right|,\left|\{\mathrm{TP}\}_{j^{*}}\right|\right\} \leq 20$. Still, neighbor macro BS for each micro BS is known from the network topology (often is no more than 3 ), so the complexity is less than $O\left(3\left|\mathbf{B}_{m}\right|+20\left|\mathbf{B}_{M}\right|\right)$, which means complexity is only determined by regional BS number.

Since this algorithm analyzes the compensatory method only from view of BS load and state, we need to consider regional and BS power constraint, coverage constraint, interference constraint, QoS constraint, and so forth. Aiming at solving optimization problem from the perspective of users, the paper designs distribution user allocation algorithm to achieve the optimal allocation for users next. 
Input: $\mathbf{B}, \mathbf{U}(t), \mathbf{X}(t), \mathbf{P}(t)$ Output: $\mathbf{X}(t), \mathbf{P}(t)$
(1) $\boldsymbol{U}(t)=\mathbf{U}(t)$
(2) while $\mathcal{U}(t) \neq \varnothing$
(3) for $\forall i^{*} \in \mathcal{U}(t), B_{j^{*}} \Leftarrow \arg _{B_{j} \in \mathbf{B}} \max \left\{\sigma_{i^{*} j}(t) \mid L_{j}(t)<1\right\}$
(4) while $\sigma_{i^{*} j^{*}}(t)<\chi$ or $\sigma_{i^{*} j^{*}}(t) /\left(\mathcal{N}_{0}+\sum_{k=1, k \neq j^{*}}^{N} P_{k}^{T}(t) g_{i^{*} k}(t)\right)<\gamma_{\min }$
(5) $\quad p_{i^{*} j^{*}}(t) \Leftarrow p_{i^{*} j^{*}}(t)+\Delta p, x_{i^{*} j^{*}}(t)=1$
(6) if $\exists k, P_{j^{*} k}^{B}(t)>P_{T}^{B}$, break; end if
(7) $\quad$ if $\sum_{i=1}^{\left|\mathbf{M}_{j^{*}}(t)\right|} \beta_{i j^{*}}(t) p_{i j^{*}}(t)>\alpha P_{j^{*}}^{T}$, break; end if
(8) if $L_{j^{*}}(t)+\beta_{i^{*} j^{*}}(t)>1$, break; end if
(9) end while
(10) $\boldsymbol{U}(t) \Leftarrow \mathcal{U}(t) \backslash\left\{i^{*}\right\}$
(11) end while

Algorithm 2: Description of distribution user allocation algorithm.

$$
\begin{aligned}
& \text { Input: } P_{j}(t), A_{j}(t), E_{j}(t) \text { Output: } A_{j}(t), E_{j}(t) \\
& \text { (1) if } E_{j}(t) \geq \int_{t}^{t+1} P_{j}(t) d t \text {, } \\
& \text { (2) else } \\
& E_{j}(t)=E_{j}(t)-\int_{t}^{t+1} P_{j}(t) d t+\int_{t}^{t+1} v_{j}(t) d t \text { and } a_{j}(t)=0 \\
& \text { (3) } E_{j}(t)=E_{j}(t)+\int_{t}^{t+1} v_{j}(t) d t \text { and } a_{j}(t)=P_{j}(t) \\
& \text { (4) end if }
\end{aligned}
$$

Algorithm 3: Description of sustainable power supply algorithm.

4.3. Distribution User Allocation Algorithm (DUAA). Above BS cooperation algorithm mainly concentrates on sleep nodes method and load reallocation. Further, user-BS association needs to consider specific user allocation. In this part, the regional power is minimized subject to the constraints in (10). The microscopic problem is a complex combinational optimization problem as well. Thus, this paper employs a lowcomplexity DUAA to solve it.

We use $\mathbf{U}(t)$ to designate the set of users at time $t$, where $\mathbf{U}(t)=\mathbf{U M}_{j}(t)$. For arbitrary user $i^{*}$ in $\mathbf{U}(t)$, select the corresponding BS $j^{*}$ with the strongest received signal. If either $\gamma_{i^{*} j^{*}}(t)$ or $\sigma_{i^{*} j^{*}}(t)$ does not meet the requirements, it can be considered that the serving BS of user $i^{*}$ is slept. And we can adjust power per $\mathrm{RB} p_{i^{*} j^{*}}(t)$ of $j^{*}$ to satisfy constraints.

According to [7], in LTE system, $I_{i j}(t)$ is generally set as 0 . Based on RB conflict principle, $\mathscr{I}_{i}(t)$ can be written as

$$
\mathscr{I}_{i}(t)=\sum_{j=1, j \neq i}^{N} L_{i}(t) L_{j}(t) P_{j}^{T}(t) g_{i j}(t) .
$$

Then we have

$$
\begin{aligned}
\gamma_{i j}(t) & =\frac{\sigma_{i j}(t)}{\mathcal{N}_{0}+\sum_{k=1, k \neq j}^{N} L_{i}(t) L_{k}(t) P_{k}^{T}(t) g_{i k}(t)} \\
& \geq \frac{\sigma_{i j}(t)}{\mathcal{N}_{0}+\sum_{k=1, k \neq j}^{N} P_{k}^{T}(t) g_{i k}(t)} .
\end{aligned}
$$

Obviously, if the latter term in (16) is not less than $\gamma_{\min }$, it can be derived that $\gamma_{i j}(t) \geq \gamma_{\min }$. Assuming that the step to adjust power is $\Delta p$, this algorithm is described in Algorithm 2. Since adjustable parameter is only power per RB allocated to each user, which is irrelevant to other users and BS load, DUAA is a distributed algorithm without centralized control.

Given that the scope of $p_{i j}$ is $\left[p_{\min }, p_{\max }\right]$ and the complexity to compute $P_{j^{*} k}^{B}(t)$ is $\Lambda$, then the complexity of DUAA is $O\left(\left(p_{\max }-p_{\min }\right) / \Delta p \cdot \Lambda \cdot K \cdot|\mathbf{B}|^{2} \cdot|\mathbf{U}(t)| \cdot \max \left\{\mathbf{M}_{j}(t)\right\}\right)$. As $K$ is always a constant and the iteration upper limit is definite when range and step of $p_{i j}$ are known, computation complexity is just $O\left(\Lambda \cdot|\mathbf{B}|^{2} \cdot|\mathbf{U}(t)|^{2}\right)$, which is an acceptable quadratic polynomial.

4.4. Sustainable Power Supply Algorithm. With above three algorithms we can obtain the BS modes, the traffic reallocation methods, and user-BS association strategies. However, they are all focusing on the power of BS requirement without considering hybrid energy supplies. Here, sustainable power supply algorithm is proposed to maximize the green energy utilization. For each eNodeB, we will execute the algorithm as Algorithm 3, which determines function $f(\cdot)$ and $h(\cdot)$. To make energy supply more stable, the energy supply method is consistent with the approach in $[13,18]$. Still, assume time interval during $t$ and $t+1$ is one hour here. Algorithm 3 will be executed at each time $t$ as well.

That is, only when the storage energy of renewable energy is higher than the eNodeB power required during the next 


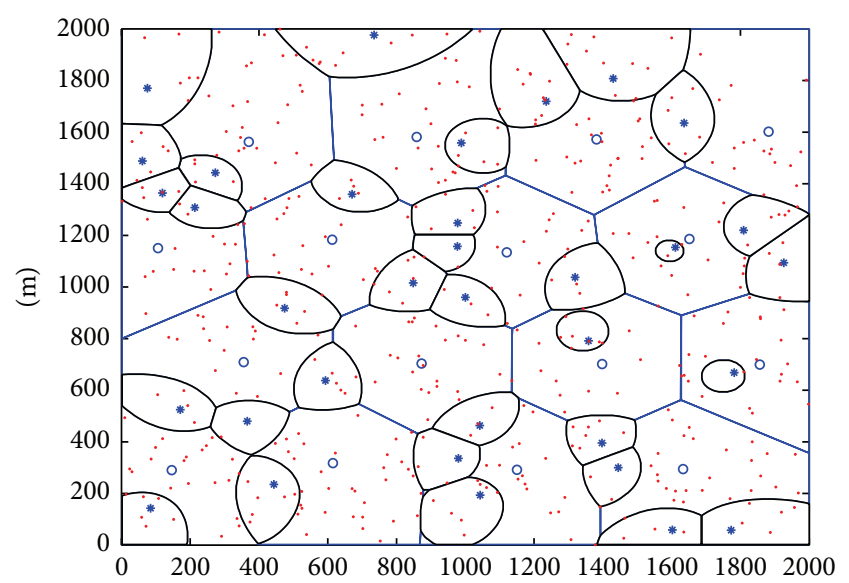

(m)

FIGURE 4: Illustration of simulation scenario.

time interval will the renewable energy be used. Otherwise, the energy will be stored for the next time intervals.

In this algorithm, as $A_{j}(t)$ and $E_{j}(t)$ just need to be computed at each time point with linear judgment for each eNodeB, so its complexity is only $O\left(\left|\mathbf{B}_{M}\right|\right)$, which is linear with eNodeB number.

\section{Simulation and Analysis}

5.1. Simulation Scenario. The simulation is performed in LTE underlay heterogeneous network scenario as illustrated in Figure 4. This part of network covers a $2000 \mathrm{~m} \times 2000 \mathrm{~m}$ square area which includes 16 eNodeBs and 34 microcells. In this figure, blue asterisks denote the locations of eNodeB, blue circles denote the locations of microcell, and red bullets denote the users at a time point. Still, we assume that users are uniformly distributed in the network, and we only consider $512 \mathrm{kbps}$ CRB services in the network. The path loss employs COST-231 Hata Model. The BS carrier frequency, penetration loss, antenna gain, and thermal noise are $2 \mathrm{GHz}, 10 \mathrm{~dB}, 10 \mathrm{~dB}$, and $-174 \mathrm{dBm} / \mathrm{Hz}$, respectively.

Moreover, for resource allocation model, the number of RBs for eNodeB and microcell is 100 and 20. The attenuation factor $\xi$ is 0.95 . And $\gamma_{\min }$ and $\gamma_{\max }$ are $-13 \mathrm{~dB}$ and $20 \mathrm{~dB}$, respectively. $\varphi_{\max }$ is $4.8 \mathrm{Mbps}$. Bandwidth of each $\mathrm{RB}$ is $180 \mathrm{KHz}$.

In BS energy consumption model and QoS evaluation model, the maximal transmit power of eNodeB and micro $\mathrm{BS}$ is $20 \mathrm{~W}$ and $10 \mathrm{~W}$, while the maximum operational power is $500 \mathrm{~W}$ and $15 \mathrm{~W}$, respectively. The ratio of static power to maximum operational power of eNodeB and microcell is supposed to be 0.8 and 0.33 . And $\varepsilon$ and power amplifier efficiency are fixed as 0.05 and 0.2 for all BSs. Primary energy of all eNodeB panels is set to be 0. Using S-ARIMA based algorithm in Section 4, for normalized traffic which comes from a city in China, we predict traffic variations for Friday as shown in Figure 5. We have found that S-ARIMA $(1,1,1) \times$ $(0,1,1)_{24}$ is the most accurate model with highest correlation coefficient, 0.996 .

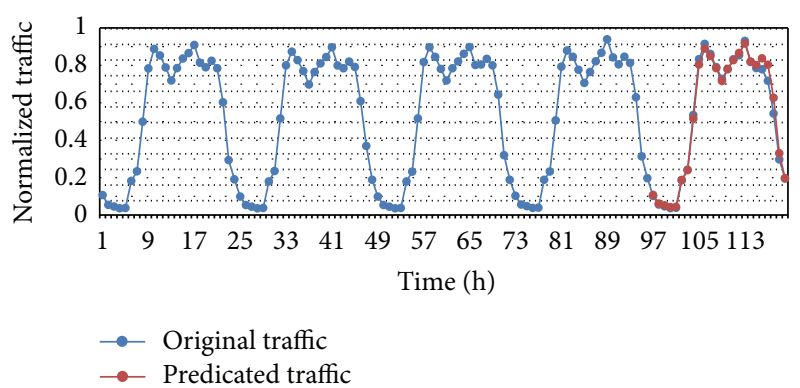

Figure 5: Traffic prediction for Friday with data from Monday to Thursday.

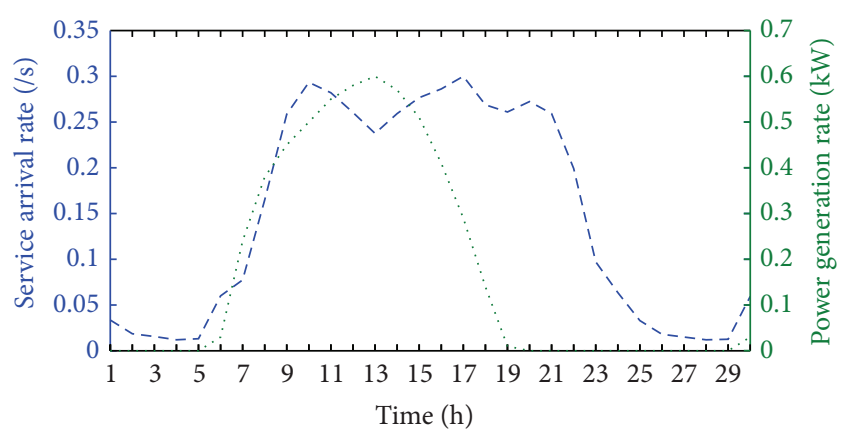

FIGURE 6: Service arrival rate and power generation rate.

TABLE 1: Simulation parameters.

\begin{tabular}{lcc}
\hline Parameter & Value & Unit \\
\hline$P_{T}^{B}$ & 1 & $\%$ \\
$\alpha$ & 0.9 & - \\
$\chi$ & -105 & $\mathrm{dBm}$ \\
$P_{\sigma}$ & 97 & $\%$ \\
$P_{\gamma}$ & 98 & $\%$ \\
$P_{\min }$ & 0.1 & Watt \\
$P_{\max }$ & 1 & Watt \\
$\Delta p$ & 0.05 & Watt \\
\hline
\end{tabular}

According to the prediction results, here we use a time period of 29 hours predicated for Friday as the simulation time. Service arrival rate in the region and energy generation rate of solar panels are depicted in Figure 6, where the average service time is 5 minutes and the number of available resource is the maximum resource number. Here, arrival rate is consistent with the predicted results and the power generation rate is the same as [18]. At the beginning of each hour, user arrives at each BS with the same Poisson arrival process as shown in Figure 6. S-ARIMA algorithm is implemented with RStudio. And the rest of the algorithms are simulated under MATLAB. The values of other parameters used in simulations are outlined in Table 1.

According to the models and parameters abovementioned, simulation results are given as follows.

5.2. Result Analysis. The simulation is performed in LTE under heterogeneous network and considers time-variant 


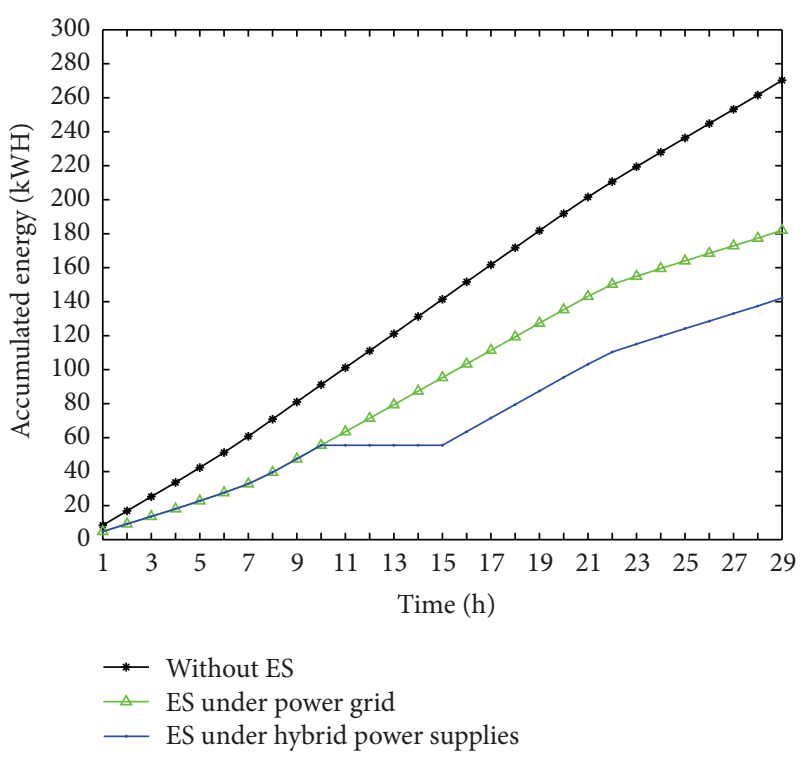

FIGURE 7: Comparison of ES performance under different mechanisms.

characters, which is less studied yet. Therefore, this paper emphasizes the analysis of ES BSs numbers, energy efficiency, and QoS, coverage, and interference parameters.

It is true that executing ES algorithms and schemes always puts additional computation and management burden of the management center, and energy consumption may increase as well. However, in our mechanism, these algorithms and schemes are mainly executed in centralized SON at OAM system and distributed SON and SON agents on the BSs. For distributed SON and SON agents on the BS mainly responsible for ES action costs, the energy costs have been taken into consideration in (5) with ratio $\varepsilon$ denoting energy proportion of sleep BSs to maintaining basic management functions. With these for active BSs with compensation actions, we can assume that the control energy costs can be accommodated by power increase. For centralized SON located at OAM system, the number of these nodes is fairly lower than number of BSs, so their energy consumption is much lesser than BSs. Besides, as we adopt algorithms and schemes with low computation complexity, their additional energy consumption is inappreciable compared to energysaving gains for BSs. Considering that these additional energies are minor and hard to be quantified, we just ignore them here.

In the whole time domain, the maximum number of sleep macro BSs is 7, and sleep time intervals are $\{2 \sim 9\}$ and $\{24 \sim$ $30\}$. In addition, all micro BSs can be slept under constraints between 11 and 34 ones for different hours. In time domain $T$, energy consumption of normal state is labeled as $F(T)$, and energy consumption of using ES method is labeled as $F^{\prime}(T)$; then ES gain in time domain $G_{E}(T)$ can be expressed as

$$
G_{E}(T)=\frac{F(T)-F^{\prime}(T)}{F(T)} \times 100 \% .
$$

Figure 7 shows the variation of regional accumulated energy consumption for three different methods, which are

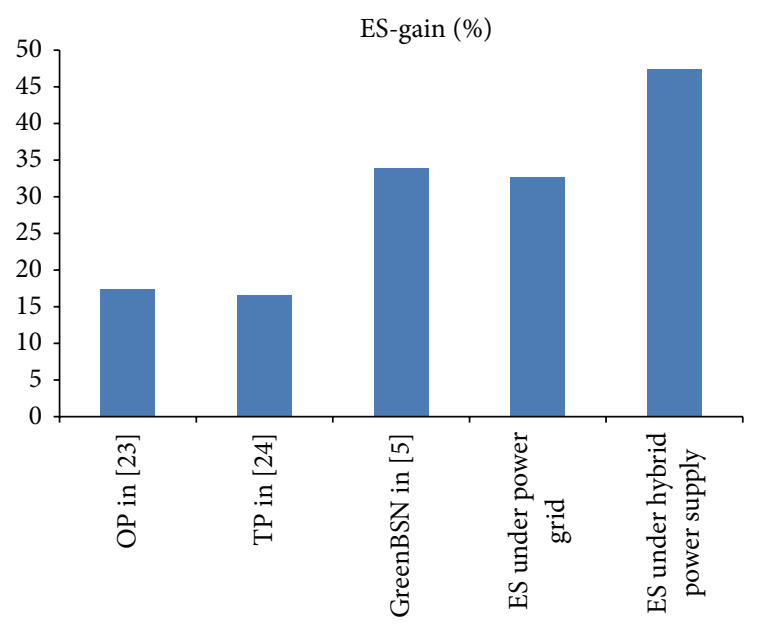

FIGURE 8: ES gain comparison for different methods.

method without ES mechanism, method with ES under power grid, and method with ES under hybrid power supplies. Here ES under power grid means only S-ARIMA, BCAGT, and DUAA are adopted, and ES under hybrid power supplies mean that all the algorithms in this paper are used. Compared with conventional method, energy consumption of power grid can be saved more with renewable energy. During time interval $\{10 \sim 15\}$, renewable energy system can satisfy energy demands individually.

As ES methods in $[23,24]$ just take ES actions once during the period, there is no doubt that ES method proposed in this paper will take on higher energy efficiency than them. As shown in Figure 8, compared with OP method in [23], TP method in [24], and classical GreenBSN in [5] (here we just assume BS radius for eNodeB uses the value in [17]), we can find that ES gains of our proposed ES mechanisms are $32.65 \%$ and $47.40 \%$, respectively, which are almost twice for OP (17.32\%) and TP (16.51\%). However, GreenBSN takes on little higher ES efficiency (33.86\%) than our ES under power grid as it is a nearly optimal method. But it is theoretical to some extent as interference control is not preferred.

Since ES mechanism has impact on system performance, in the following, we analyze coverage, interference, and QoS indicators, respectively. There is no doubt that our mechanism is worse than methods in $[23,24]$ as more BSs are slept. So here we mainly explore the performance of our mechanism after execution.

To evaluate performance effect of our algorithm, we choose the time point with most sleep BSs (which is the 29th hour) and analyze the RSRP and SINR distributions for the active eNodeB with highest traffic load at this time point. Figure 9 shows cumulative probability distribution of coverage indicator RSRP for the selected BS. As DUAA just considers power control for users under acceptable levels, coverage and interference effects for other active users should be evaluated as well. Here ES (users) means performance for user set whose power has been adjusted through DUAA, and ES (regional) means performance for all the active users in this network. It can be seen that ES mechanism degrades coverage 


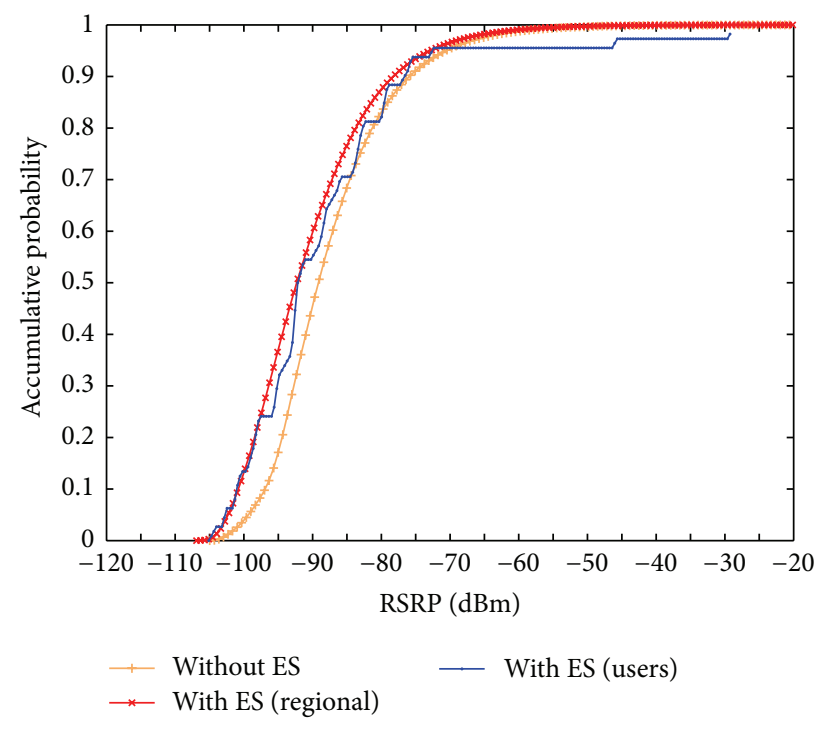

FIGURE 9: Cumulative probability distribution of RSRP.

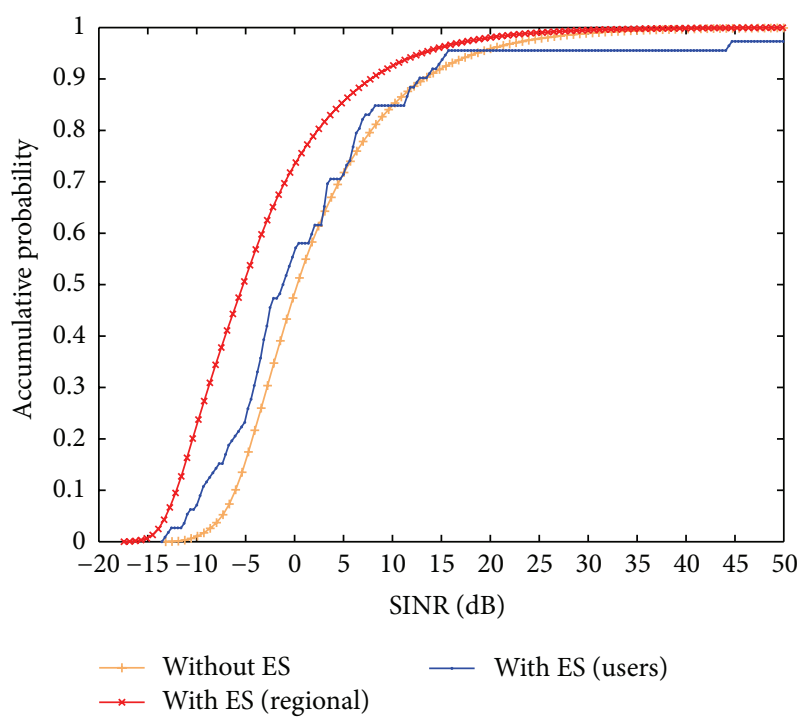

FIGURE 10: Cumulative probability distribution of SINR.

performance to some extent. In the analysis, we consider the effect on active users as well as effect on overall coverage performance of selected BS. Because our mechanism mainly emphasizes power control for active users under sleep BSs, so RSRP cumulative probability distribution of active users is generally better than all the users in the network. Further, cumulative probabilities for active users and regional RSRP (more than $-105 \mathrm{dBm}$ ) are both $100 \%$, which proves that coverage performance conforms to constraints.

Similarly, from the perspective of interference, cumulative probability distribution of interference indicator for selected BS is illustrated in Figure 10. We can see that ES mechanism can negatively affect regional interference as well. Moreover, SINR cumulative probability distribution of active users also performs better than SINR distribution of overall coverage. Meanwhile, cumulative probabilities of SINR (more

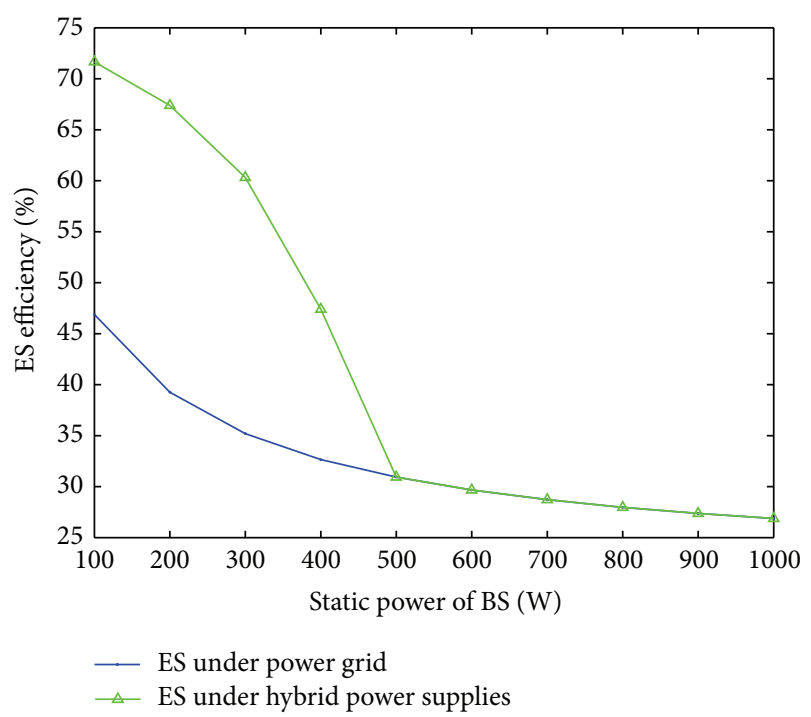

FIGURE 11: Regional ES gain with static power variation per BS.

than $-105 \mathrm{dBm}$ ) for active users under sleep BSs and for all the users in the network are $100 \%$ and $98.1 \%$, respectively, which means interference meets constraints as well.

As for QoS, with computation method in [25], simulation results indicate that maximum service blocking probability is less than the target $1 \%$, which indicates that it satisfies QoS constraint.

In order to verify scalability, ES efficiency for BSs with different static powers is further studied under simulation scenario. As shown in Figure 11, on the premise that sleep node method is determined, ES efficiency decreases as BS static power increases, which shows that BS static power is bottleneck of ES efficiency. In other words, reducing BS static power can enhance energy efficiency significantly. When BS static power is lower than 500 Watt, regional energy consumption is less. Thus, it can be powered by renewable energy. At this point, the ES mechanism mentioned in this paper performs much better than conventional sleep node methods. When BS static power is equal to 100 Watt, both mechanisms can achieve optimal energy gains, which are $71.66 \%$ and $46.88 \%$, respectively. Conversely, when BS static power is more than or equal to 500 Watt, regional energy consumption is more than available renewable energy, which means only power grid can be used. Thus, ES effects of two methods tend to be the same and reach the peak efficiency $30.93 \%$ at 500 Watt. It indicates that renewable energy has certain limitations because of its low generation rate.

Consequently, the mechanism can reduce energy consumption of LTE heterogeneous network while maintaining satisfactory coverage, interference, and QoS. In addition, it can implement efficient ES for BSs with different power, thereby having strong adaptability.

\section{Conclusion}

For LTE heterogeneous network, this paper proposes an ESM mechanism based on hybrid energy supplies. With 
simulations under irregular topology in LTE underlay heterogeneous network, this paper verifies that this mechanism can save $47.4 \%$ energy while ensuring the acceptable regional coverage, interference, and QoS and has strong adaptability. In our further study, we can take into account new characters of LTE/LTE-A network. Moreover, new technologies such as CoMP, Relay, and D2D can be used to achieve regional compensation, thereby implementing ES, reducing interference, and enhancing resource utilization. Additionally, some innovative indicators, such as power per bit and power per square, can be set as optimization objectives to construct ES models. Still, energy pool technologies which can share the renewable energy among different BS will be studied. Wireless powering and energy-harvesting technologies for BS power supply will be considered as well.

\section{Competing Interests}

The authors declare that they have no competing interests.

\section{Acknowledgments}

This research is supported by the National High Technology Research and Development Program of China (2015AA01A705) and Natural Science Foundation of China (61271187).

\section{References}

[1] K. Davaslioglu and E. Ayanoglu, "Quantifying potential energy efficiency gain in green cellular wireless networks," IEEE Communications Surveys \& Tutorials, vol. 16, no. 4, pp. 2065-2091, 2014.

[2] E. Oh, K. Son, and B. Krishnamachari, "Dynamic base station switching-on/off strategies for green cellular networks," IEEE Transactions on Wireless Communications, vol. 12, no. 5, pp. 2126-2136, 2013.

[3] J. Wu, Y. Zhang, M. Zukerman, and E. K.-N. Yung, "Energyefficient base-stations sleep-mode techniques in green cellular networks: a survey," IEEE Communications Surveys and Tutorials, vol. 17, no. 2, pp. 803-826, 2015.

[4] A. Kumar and C. Rosenberg, "Energy and throughput tradeoffs in cellular networks using base station switching," IEEE Transactions on Mobile Computing, vol. 15, no. 2, pp. 364-376, 2016.

[5] C. Peng, S.-B. Lee, S. Lu, and H. Luo, "GreenBSN: enabling energy-proportional cellular base station networks," IEEE Transactions on Mobile Computing, vol. 13, no. 11, pp. 2537-2551, 2014.

[6] Z. Niu, X. Guo, S. Zhou, and P. R. Kumar, "Characterizing energy-delay tradeoff in hyper-cellular networks with base station sleeping control," IEEE Journal on Selected Areas in Communications, vol. 33, no. 4, pp. 641-650, 2015.

[7] M. F. Hossain, K. S. Munasinghe, and A. Jamalipour, "Energyaware dynamic sectorization of base stations in multi-cell ofdma networks," IEEE Wireless Communications Letters, vol. 2, no. 6, pp. 587-590, 2013.

[8] J. Peng, P. Hong, and K. Xue, "Stochastic analysis of optimal base station energy saving in cellular networks with sleep mode," IEEE Communications Letters, vol. 18, no. 4, pp. 612-615, 2014.
[9] N. Deng, M. Zhao, J. Zhu, and W. Zhou, "Traffic-aware relay sleep control for joint macro-relay network energy efficiency," Journal of Communications and Networks, vol. 17, no. 1, pp. 4757, 2015.

[10] L. Suárez, L. Nuaymi, and J.-M. Bonnin, "Energy-efficient BS switching-off and cell topology management for macro/femto environments," Computer Networks, vol. 78, pp. 182-201, 2015.

[11] S. Morosi, P. Piunti, and E. Del Re, "Sleep mode management in cellular networks: a traffic based technique enabling energy saving," Transactions on Emerging Telecommunications Technologies, vol. 24, no. 3, pp. 331-341, 2013.

[12] D. Paolo, M. Marco, B. Nicola, and B. Nicola, "A model to analyze the energy savings of base station sleep mode in LTE HetNets," in Proceedings of the IEEE International Conference on and IEEE Cyber, Physical and Social Computing and Internet of Things Green Computing and Communications (GreenCom '13), pp. 1375-1380, Beijing, China, August 2013.

[13] T. Han and N. Ansari, "On optimizing green energy utilization for cellular networks with hybrid energy supplies," IEEE Transactions on Wireless Communications, vol. 12, no. 8, pp. 3872 3882, 2013.

[14] D. Zordan, M. Miozzo, P. Dini, and M. Rossi, "When telecommunications networks meet energy grids: cellular networks with energy harvesting and trading capabilities," IEEE Communications Magazine, vol. 53, no. 6, pp. 117-123, 2015.

[15] J. Gong, J. S. Thompson, S. Zhou, and Z. Niu, "Base station sleeping and resource allocation in renewable energy powered cellular networks," IEEE Transactions on Communications, vol. 62, no. 11, pp. 3801-3813, 2014.

[16] 3GPP, "Energy Saving Management (ESM); concepts and requirements," 3GPP TS 32.551, Version 11.3.0, 2012.

[17] P. Yu, L. Feng, Z. Li, W. Li, and X. Qiu, "Low-complexity energy efficient base station cooperation mechanism in LTE networks," KSII Transactions on Internet and Information Systems, vol. 9, no. 10, pp. 3921-3944, 2015.

[18] P. Yu, J.-P. Cao, S.-X. Zhang, and W.-J. Li, "Energy-saving management mechanism based on hybrid energy supplies for wireless cellular networks," Journal of Beijing University of Posts and Telecommunications, vol. 38, no. 1, pp. 46-50, 2015.

[19] M. F. Hossain, K. S. Munasinghe, and A. Jamalipour, "On the energy efficiency of self-organizing LTE cellular access networks," in Proceedings of the IEEE Global Communications Conference (GLOBECOM '12), pp. 5314-5319, IEEE, Anaheim, Calif, USA, December 2012.

[20] N. Saxena, B. J. R. Sahu, and Y. S. Han, "Traffic-aware energy optimization in green LTE cellular systems," IEEE Communications Letters, vol. 18, no. 1, pp. 38-41, 2014.

[21] M. Deruyck, E. Tanghe, W. Joseph, and L. Martens, "Modelling and optimization of power consumption in wireless access networks," Computer Communications, vol. 34, no. 17, pp. 20362046, 2011.

[22] M. F. Hossain, K. S. Munasinghe, and A. Jamalipour, "On the eNB-based energy-saving cooperation techniques for LTE access networks," Wireless Communications and Mobile Computing, vol. 15, no. 3, pp. 401-420, 2015.

[23] P. Yu, W.-J. Li, and X.-S. Qiu, "A regional autonomic energysaving management mechanism for cellular networks," Journal of Electronics \& Information Technology, vol. 34, no. 11, pp. 27072714, 2012.

[24] P. Yu, W. Li, and X. Qiu, "Self-organizing energy-saving management mechanism based on pilot power adjustment in 
cellular networks," International Journal of Distributed Sensor Networks, vol. 2012, Article ID 721957, 13 pages, 2012.

[25] L. Chiaraviglio, D. Ciullo, M. Meo, and M. A. Marsan, "Energyefficient management of UMTS access networks," in Proceedings of the 21st International Teletraffic Congress (ITC 21 '09), pp. 1-8, Paris, France, September 2009. 

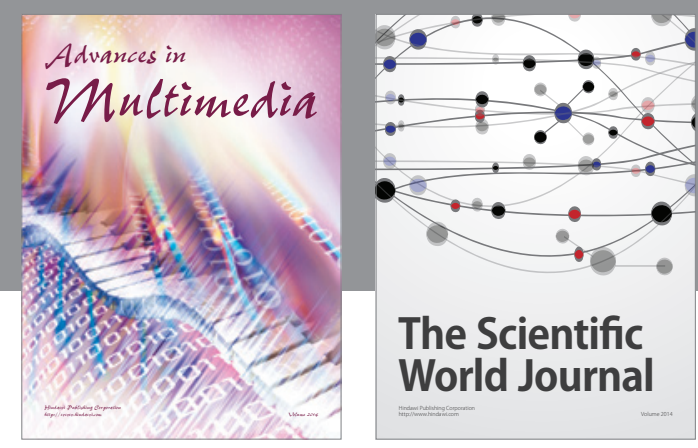

The Scientific World Journal
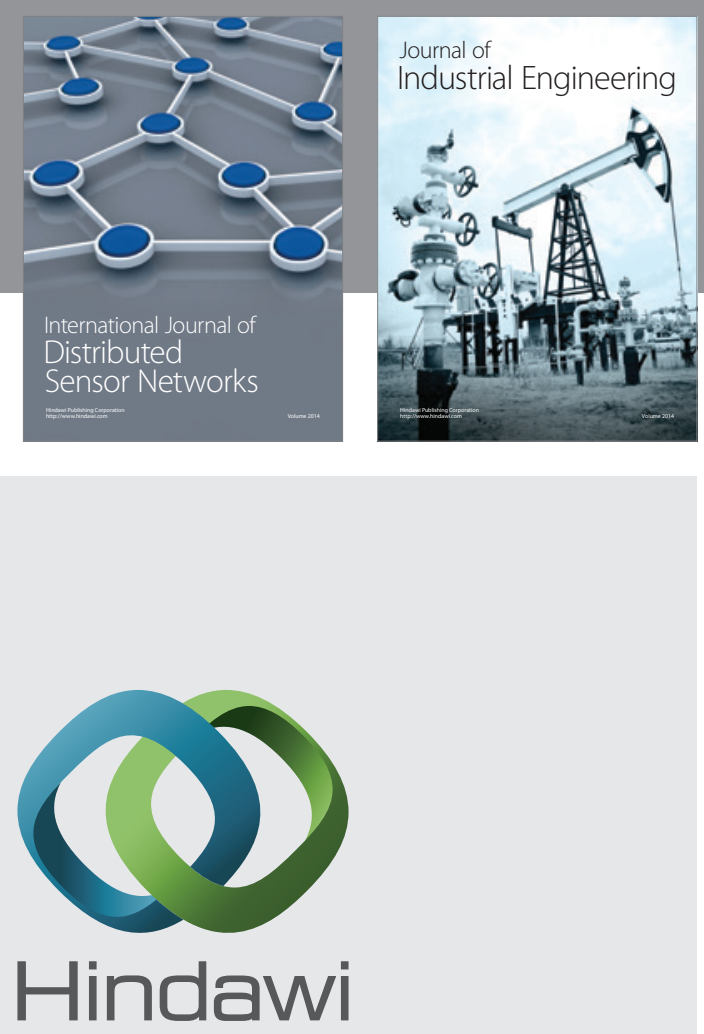

Submit your manuscripts at

http://www.hindawi.com

\section{Computer Networks} and Communications
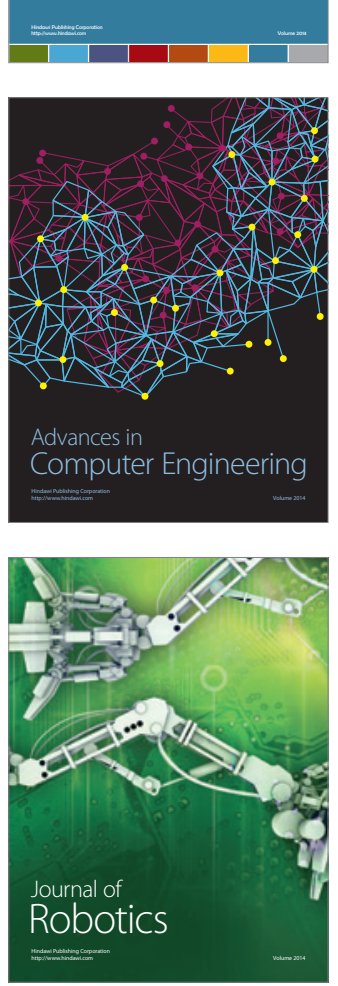
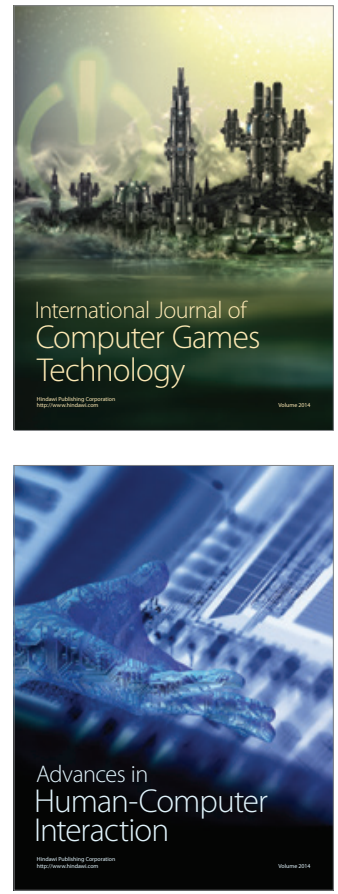
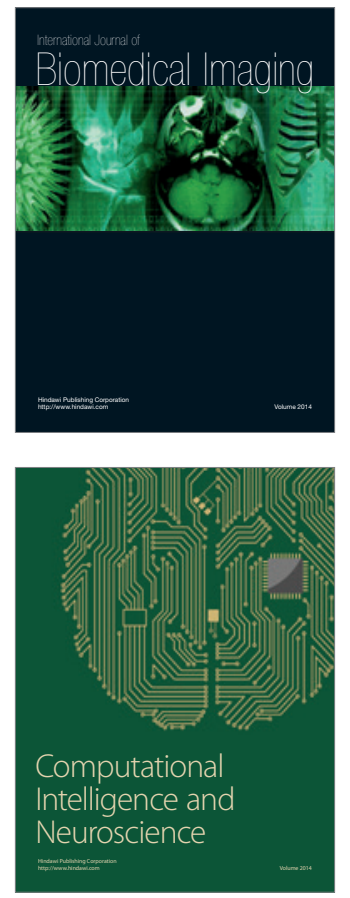
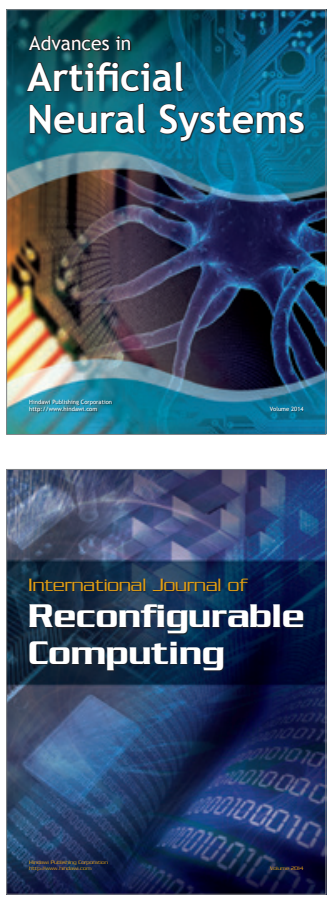
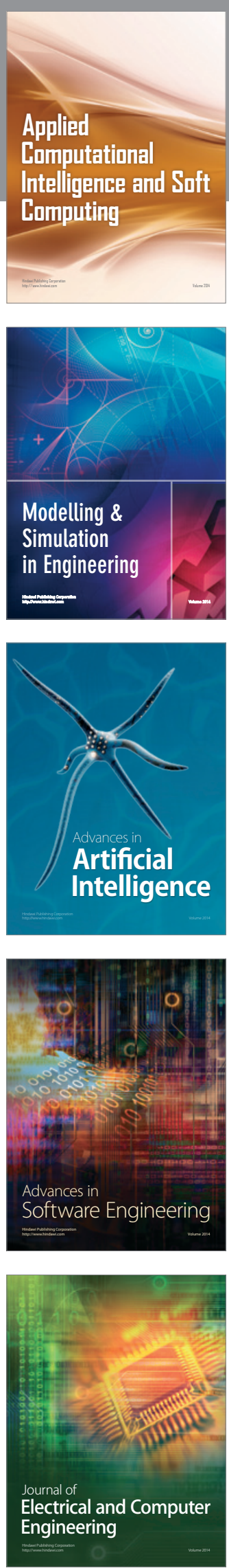